\title{
Exérese do seg'mento vertical do canalículo lacrimal na síndrome do olho seco: Estudo preliminar
}

\author{
Removal of the vertical portion of the lacrimal canaliculus in dry eye syndrome
}

Eliana Forno ${ }^{1}$

Flavio Buzalaf ${ }^{2}$
Trabalho realizado no Setor de Plástica Ocular da Divisão de Clínica Oftalmológica do Hospital das Clínicas da Faculdade de Medicina da Universidade de São Paulo - USP.

${ }^{1}$ Médica Colaboradora do Setor de Plástica Ocular do Hospital das Clínicas da Faculdade de Medicina da Universidade de São Paulo - USP - São Paulo (SP).

${ }^{2}$ Médico Estagiário do Setor de Plástica Ocular do Hospital das Clínicas da Faculdade de Medicina da USP.

Endereço para Correspondência: Eliana A. Forno

Al. Itú, 1209 Apto 93 - São Paulo (SP) CEP 01421-001

E-mail: eliana.forno@uol.com.br

Recebido para publicação em 02.07.2003

Versão revisada recebida em 13.10.2004

Aprovação em 07.01.2005

\begin{tabular}{|l|}
\hline RESUMO \\
\hline Objetivo: Avaliar a eficácia e possíveis complicações da remoção do seg- \\
mento vertical do canalículo lacrimal, em pacientes com síndrome do olho \\
seco grave. Métodos: Seis canalículos de quatro pacientes, 3 dos quais \\
tinham o diagnóstico de olho seco associado à síndrome de Sjögren primária \\
e o quarto, ceratoconjuntivite sicca por remoção completa de glândula \\
lacrimal, foram submetidos a exérese do segmento vertical do canalículo \\
lacrimal. Os critérios de inclusão foram: sinais e sintomas de olho seco que \\
não melhoraram com tratamento clínico, Schirmer menor que 5 mm, rosa \\
bengala corando córnea e conjuntiva e casos de recanalização após eletro- \\
cauterização dos pontos. Os pontos lacrimais foram avaliados por exame \\
biomicroscópico após 7, 15, 30, 90 e 180 dias da cirurgia. Resultados: No \\
período de seguimento, nenhum canalículo sofreu recanalização. Em cinco \\
olhos, houve diminuição da ceratite ponteada difusa e dos filamentos \\
corneanos e melhora nos valores do teste de Schirmere rosabengala. No olho \\
submetidoàremoção completa da glândula lacrimal, a córnea ainda apresen- \\
tava ceratite ponteada difusa, mesmo após dois meses de cirurgia. Não \\
houve alterações da margem palpebral. Conclusão: Esta técnica, além de \\
mostrar-se efetivae simples para oclusão permanente do canalículolacrimal, \\
não cursou com complicações observadas em outros procedimentos. \\
\hline
\end{tabular}

Descritores: Síndromes do olho seco/cirurgia; Aparelho lacrimal/cirurgia; Procedimentos cirúrgicos oftalmológicos/métodos

\section{INTRODUÇÃO}

A síndrome do olho seco ou ceratoconjuntivite sicca representa um grupo de doenças que resultam em alterações crônicas do filme lacrimal e da superfície ocular. Os sinais clássicos são: hiperemia conjuntival, erosões ponteadas superficiais, placas mucosas, podendo-se também observar, nos casos mais graves, ceratite filamentar e defeitos corneanos ${ }^{(1)}$. Os sintomas podem variar desde desconforto leve com ardor ocular e sensação de corpo estranho, até fotofobia e borramento visual ${ }^{(2)}$.

Há cinco causas de olho seco: por deficiência aquosa (síndrome de Sjögren, lesão do nervo lacrimal, extirpação da glândula, radioterapia, medicamentos antidepressivos, antihistamínicos, betabloqueadores, diuréticos), por deficiência de muco (penfigóide ocular, queimadura química, tracoma), deficiência lipídica (blefarite, ausência congênita da glândula de meibomius), por epiteliopatia (ceratite neuroparalítica, herpética) e má posição palpebral (ectrópio, lagoftalmo) $)^{(3)}$.

A frequiência desta condição aumenta com a idade e é encontrada em até $10 \%$ de todos os adultos e $18 \%$ dos idosos ${ }^{(4)}$. Estudo feito com idosos ame- 
ricanos, também mostrou que esta doença é comum naquela população; no entanto, não há associação com sexo ou raça ${ }^{(5)}$.

Muito se fala da prevalência do olho seco em mulheres na pós-menopausa ${ }^{(1,6)}$. Em um estudo de Coorte, do qual participaram 25.665 mulheres usuárias da Terapia de Reposição Hormonal (TRO), os autores observaram as seguintes probabilidades para desenvolver aquela doença: 1,69 para mulheres que usavam apenas estrógenos e 1,29 para aquelas que associavam estrógeno e progestágeno e concluíram que o risco de desenvolver a síndrome do olho seco é alto na pós-menopausa ${ }^{(6)}$.

A propedêutica envolve: anamnese (sintomas característicos), biomicroscopia, medida do menisco lacrimal, teste com fluoresceína e rosa bengala (áreas desepitelizadas e desvitalizadas do epitélio corneano), observação do tempo de ruptura do filme lacrimal e teste de Schirmer. Na suspeita de quadro sistêmico associado, é prudente realizar testes reumatológicos (fator reumatóide, anticorpo antinuclear, anti-SSA e antiSSB) e citologia conjuntival (estratificação do epitélio e deterioração das células caliciformes), tanto na síndrome de Sjögren primária, sem envolvimento do tecido conectivo, quanto na secundária ${ }^{(7)}$.

O tratamento do olho seco se inicia com medidas clínicas (uso de lágrimas artificiais). Quando a resposta ao tratamento é insuficiente, os pontos lacrimais podem ser ocluídos temporariamente ou de maneira definitiva. Opta-se pela oclusão definitiva nos casos de olho seco severo e irreversível.

O objetivo deste trabalho é analisar uma técnica de oclusão definitiva de um dos canalículos usando uma técnica relativamente simples.

\section{MÉTODOS}

Seis olhos de quatro pacientes, três com síndrome de Sjögren primária e um submetido à extração da glândula lacrimal por tumor maligno, foram incluídos no presente estudo. Três eram do sexo feminino e um do masculino e a média de idade entre as mulheres era de 55 anos (variando de 38 a 70 anos) e o único homem do grupo tinha 47 anos. Duas pacientes estavam no período de pós-menopausa há cinco e quinze anos e faziam uso de terapia de reposição hormonal. O quadro de olho seco foi estabelecido da seguinte maneira: 1) história prévia de tratamentos anteriores que incluíram uso de lágrimas artificiais, oclusão do ponto com tampão de silicone, cauterização do ponto por eletrocautério e ressecção completa da glândula. Dois deles também aplicavam agentes mucolíticos como $\mathrm{N}$ acetilcisteína a $10 \%$ pela grande quantidade de muco. 2) queixa atual, que incluiu os seguintes sintomas: sensação de corpo estranho, queimação ocular, blefaroespasmo secundário à irritação ocular e fotofobia e 3) biomicroscopia mostrando debris no filme lacrimal, vasodilatação conjuntival e alterações epiteliais; teste com fluoresceína e rosa bengala a $1 \%$ corando córnea e conjuntiva na região da fenda palpebral, tempo de rompimento do filme lacrimal menor que 10 segun- dos e teste de Schirmer menor que $5 \mathrm{~mm}$. O critério usado para o diagnóstico da síndrome de Sjögren primária foi aplicado anteriormente $^{(8)}$ e incluiu: teste de Schirmer sem anestesia, rosa bengala, presença de um ou mais anticorpos séricos (fator reumatóide, anticorpo antinuclear, anti SS-A ou anti SSB), xerostomia e ausência da doença do tecido conectivo.

Dois pacientes sofreram cirurgia em ambos os olhos, um nos dois pontos superiores (os inferiores estavam ocluídos por cirurgia prévia) e outro paciente nos dois inferiores, sendo que este apresentava antecedente de extrusão bilateral de tampão de silicone. Nos outros dois, o procedimento foi realizado em apenas um dos olhos. Em um paciente, no ponto inferior, pelo fato do olho contralateral ter o ponto cicatrizado em decorrência de processo crônico e no paciente no qual a glândula havia sido removida, no ponto superior. Após a cirurgia, todos os pacientes foram avaliados em 7, 15, 30, 90 e 180 dias. Em todas as visitas questionou-se sobre o conforto ocular, presença ou não de epífora, melhora ou não da fotofobia e se observavam hiperemia conjuntival no final do dia.

\section{TÉCNICA CIRÚRGICA}

Anestesia: hidrocloreto de lidocaína a 2\% com epinefrina é injetada na conjuntiva tarsal e na margem palpebral (terço medial da pálpebra). Dilata-se o ponto lacrimal e com uma pinça de dente delicada apreende-se o mesmo. Com um bisturi de lâmina 11, incisa-se todo contorno do ponto lacrimal até deixá-lo livre dos tecidos que o cercam (Figura 1). A partir daí, estende-se a dissecção até o canalículo vertical, separando-o dos tecidos adjacentes. Com estas estruturas (ponto, papila e canalículo) livres e presas pela pinça, realiza-se a ressecção das mesmas. A seguir, o espaço livre deixado por elas é suturado com fio absorvível (Vicryl 6,0 ou 7,0), passado em "U". A sutura é realizada passando-se a agulha inicialmente da margem externa (pele) para a interna (conjuntiva), exteriorizando-a. A seguir, faz-se o trajeto inverso, da margem interna para a externa. Com as duas extremidades do fio voltadas para o mesmo lado, anoda-se o mesmo. Duas ou mais suturas são necessárias até as margens estarem bem coaptadas e deverão ser mantidas em posição por aproximadamente três semanas. Pomada de antibiótico tópico é aplicada no local 2 a 3 vezes ao dia por sete dias e compressas com soro fisiológico gelado aplicadas se necessário (Figuras 1, 2a, 2b e 3).

\section{RESULTADOS}

Todos os pacientes referiram diminuição da sensação de corpo estranho e da queimação ocular já no sétimo dia pósoperatório, com exceção daquele submetido à retirada da glândula lacrimal, cuja melhora foi notada no sexto mês após a oclusão do ponto. Todos os pacientes continuaram a usar lubrificantes, porém com menor frequiência. Com relação à foto- 


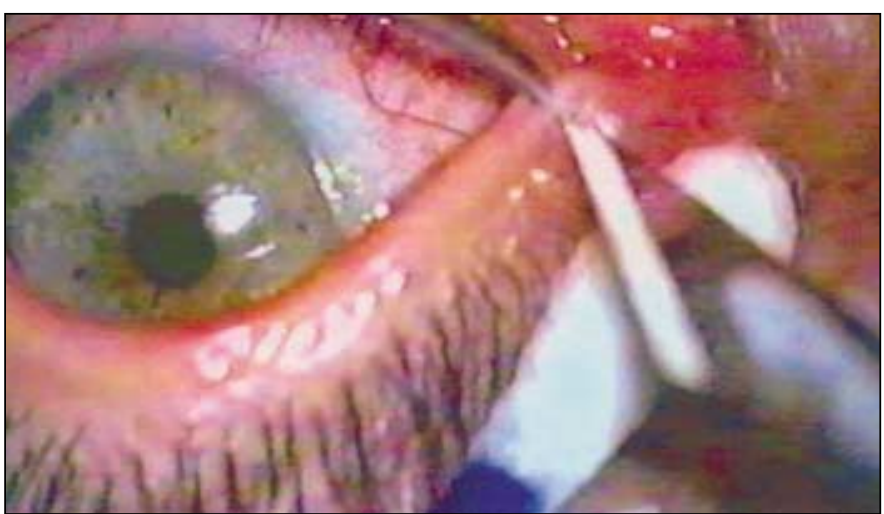

Figura 1 - Apreensão da papila com pinça de dente e incisão de todo seu contorno com lâmina 11
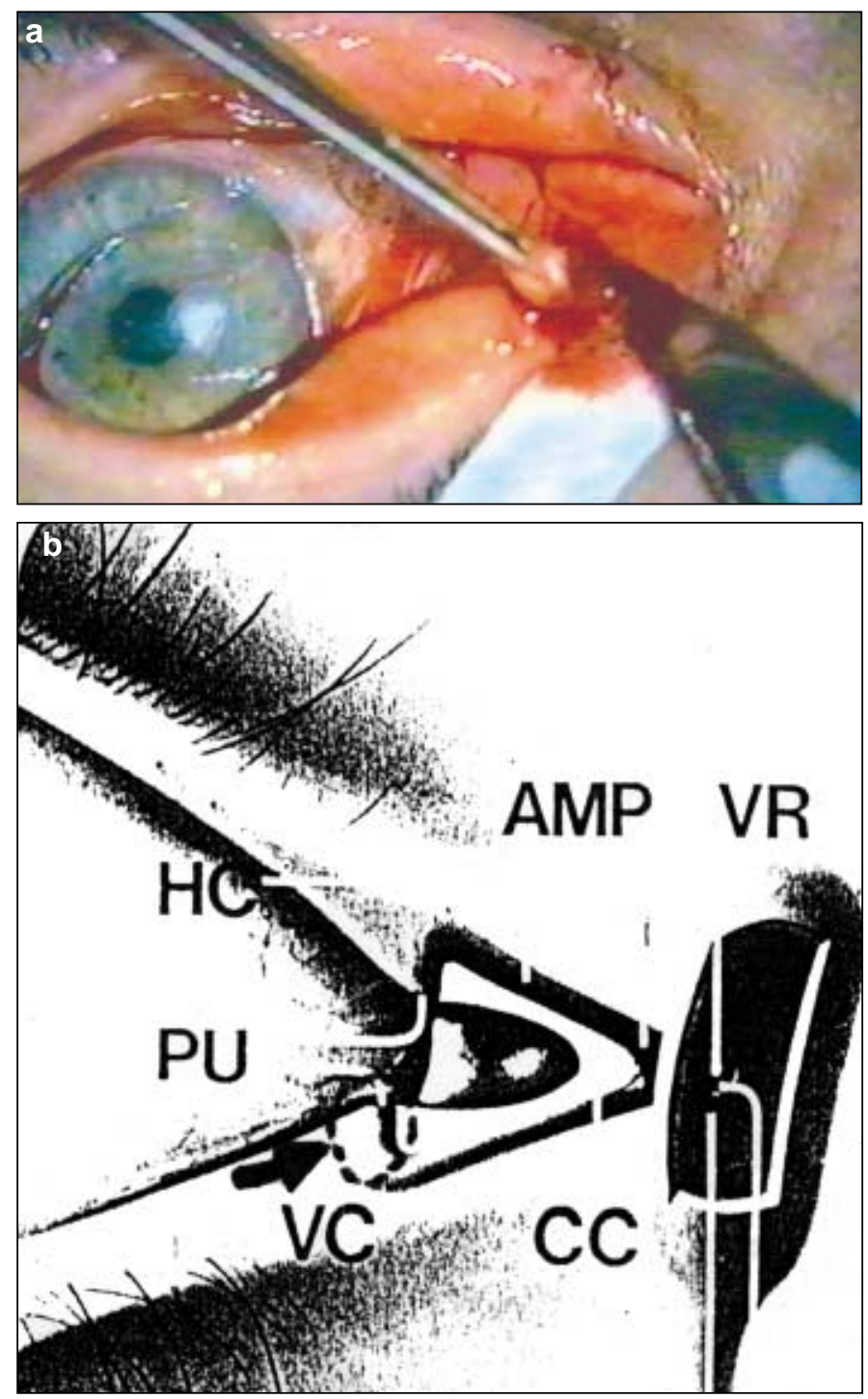

Figura 2 - a) Papila, canalículo vertical e parte da ampola livres dos tecidos adjacentes e prontos para serem ressecados. 0 espaço "morto" deixado por eles é suturado com pontos em "U" com fio absorvível; b) O pontilhado mostra o local a ser excisado. PU: Pontos lacrimais; VC: Canalículo vertical; AMP: Ampola; HC: Canalículo horizontal; CC: Canalículo comum; VR: Válvula de Rosenmuller

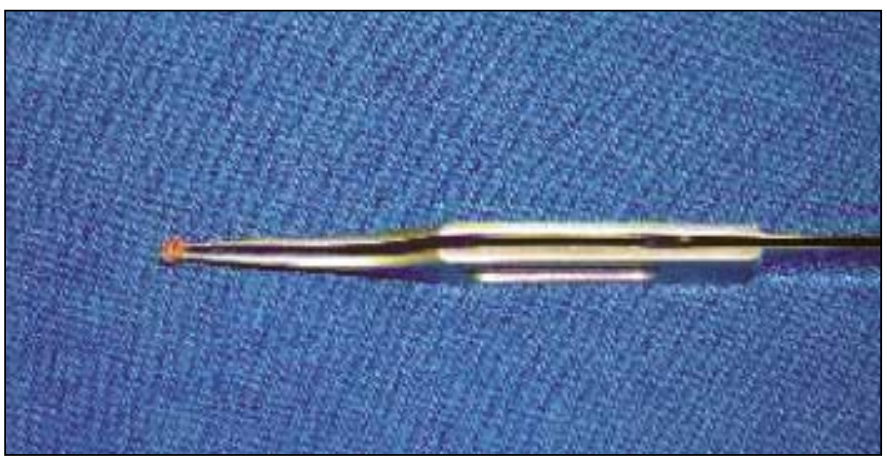

Figura 3 - Figura mostrando o segmento do canalículo removido

fobia, houve melhora nos dois pacientes que a relatavam antes do procedimento, embora este sintoma tenha sido mais expressivo em um deles. A vermelhidão ocular presente em todos os casos antes do procedimento, apresentou-se no final do acompanhamento, apenas como uma vasodilatação mínima.

Na biomicroscopia, observou-se boa evolução dos sinais, tanto em relação à ceratite ponteada, quanto aos filamentos corneanos. Foi possível observar diminuição da coloração pelo rosa bengala na região interpalpebral.

A melhora nos valores do teste de Schirmer e tempo de ruptura do filme lacrimal também foram evidentes. Quanto ao primeiro, todos os olhos com resultados pré-operatórios menores que $5 \mathrm{~mm}$, cursaram com valores pós-operatórios de 7 a $9 \mathrm{~mm}$ (média de $8,16 \mathrm{~mm}$ ) no primeiro trimestre e de 9 a $12 \mathrm{~mm}$ (média de $10 \mathrm{~mm}$ ) no segundo trimestre. Em relação ao segundo, um tempo maior que oito segundos (média de 8,5 s) foi obtido em três pacientes, no terceiro mês e maior que onze segundos (média de $12 \mathrm{~s}$ ) no sexto.

Nenhum indivíduo apresentou recanalização dos pontos operados ou alterações na margem palpebral até o final do acompanhamento. A descrição detalhada dos resultados de cada caso está listada na tabela 1.

\section{DISCUSSÃO}

O efeito da oclusão definitiva dos canalículos lacrimais na síndrome do olho seco e as possíveis complicações deste procedimento foram investigadas após se ter usado uma técnica cirúrgica simples e nunca antes empregada. Esta técnica, na qual realizou-se a excisão do segmento vertical do canalículo, foi idealizada no sentido de se obter uma oclusão permanente dos canalículos lacrimais em casos de portadores de ceratoconjuntivite sicca grave.

A canaliculectomia descrita por Putterman, se diferencia da técnica aqui apresentada, uma vez que utiliza uma sonda guia de Bowman 2-0 que deve ser introduzida até o saco lacrimal. A partir daí, uma incisão é feita na conjuntiva e na parede posterior do canalículo, sobre a sonda, desde o ponto lacrimal até o canto medial, dissecando-se toda a parede do ponto e do canalículo (segmentos vertical e horizontal), extirpando-os por completo ${ }^{(9)}$. 


\begin{tabular}{|c|c|c|c|c|c|c|c|c|c|}
\hline \multirow[t]{2}{*}{ Sexo } & \multirow{2}{*}{$\begin{array}{l}\text { Idade } \\
\text { (anos) }\end{array}$} & \multirow[t]{2}{*}{ Diagnóstico } & \multirow{2}{*}{$\begin{array}{l}\text { Canalículo } \\
\text { ocluído }\end{array}$} & \multirow{2}{*}{$\begin{array}{l}\text { Quadro } \\
\text { clínico }\end{array}$} & \multicolumn{5}{|c|}{ Evolução do quadro clínico } \\
\hline & & & & & $7^{\circ}$ dia & $15^{\circ} \mathrm{dia}$ & $30^{\circ}$ dia & $90^{\circ}$ dia & $180^{\circ}$ dia \\
\hline$F$ & 38 & $\begin{array}{l}\text { Síndrome } \\
\text { de Sjögren } \\
\text { primária }\end{array}$ & $\begin{array}{l}\text { Canalículo } \\
\text { inferior } \\
\text { (ponto inferior } \\
\text { contralateral } \\
\text { cicatriz) }\end{array}$ & $\begin{array}{l}\text { Sensação de corpo estranho } \\
\text { Ardor } \\
\text { Fotofobia } \\
\text { Hiperemia } \\
\text { Recanalização }\end{array}$ & $\begin{array}{l}\text { Melhora } \\
\text { Melhora } \\
\text { Presente } \\
\text { Presente } \\
\text { Ausente }\end{array}$ & $\begin{array}{l}\text { Melhora } \\
\text { Melhora } \\
\text { Melhora } \\
\text { Melhora } \\
\text { Ausente }\end{array}$ & $\begin{array}{l}\text { Melhora } \\
\text { Melhora } \\
\text { Melhora } \\
\text { Melhora } \\
\text { Ausente }\end{array}$ & $\begin{array}{l}\text { Melhora } \\
\text { Melhora } \\
\text { Melhora } \\
\text { Melhora } \\
\text { Ausente }\end{array}$ & $\begin{array}{l}\text { Ausente } \\
\text { Ausente } \\
\text { Ausente } \\
\text { Melhora } \\
\text { Ausente }\end{array}$ \\
\hline $\mathrm{F}$ & 57 & $\begin{array}{l}\text { Síndrome } \\
\text { de Sjögren } \\
\text { primária }\end{array}$ & $\begin{array}{l}\text { Canalículos } \\
\text { superiores AO } \\
\text { ( } 2 \text { pontos infe- } \\
\text { riores ocluídos } \\
\text { anteriormente) }\end{array}$ & $\begin{array}{l}\text { Sensação de corpo estranho } \\
\text { Ardor } \\
\text { Fotofobia } \\
\text { Hiperemia } \\
\text { Recanalização }\end{array}$ & $\begin{array}{l}\text { Melhora } \\
\text { Melhora } \\
\text { Presente } \\
\text { Presente } \\
\text { Ausente }\end{array}$ & $\begin{array}{l}\text { Melhora } \\
\text { Melhora } \\
\text { Presente } \\
\text { Melhora } \\
\text { Ausente }\end{array}$ & $\begin{array}{l}\text { Melhora } \\
\text { Melhora } \\
\text { Melhora } \\
\text { Melhora } \\
\text { Ausente }\end{array}$ & $\begin{array}{l}\text { Melhora } \\
\text { Melhora } \\
\text { Melhora } \\
\text { Melhora } \\
\text { Ausente }\end{array}$ & $\begin{array}{l}\text { Ausente } \\
\text { Ausente } \\
\text { Ausente } \\
\text { Melhora } \\
\text { Ausente }\end{array}$ \\
\hline $\mathrm{F}$ & 70 & $\begin{array}{l}\text { Síndrome } \\
\text { de Sjögren } \\
\text { primária }\end{array}$ & $\begin{array}{l}\text { Canalículos } \\
\text { inferiores AO }\end{array}$ & $\begin{array}{l}\text { Sensação de corpo estranho } \\
\text { Ardor } \\
\text { Fotofobia } \\
\text { Hiperemia } \\
\text { Recanalização }\end{array}$ & $\begin{array}{l}\text { Melhora } \\
\text { Melhora } \\
\text { Ausente } \\
\text { Presente } \\
\text { Ausente }\end{array}$ & $\begin{array}{l}\text { Melhora } \\
\text { Melhora } \\
\text { Ausente } \\
\text { Presente } \\
\text { Ausente }\end{array}$ & $\begin{array}{l}\text { Melhora } \\
\text { Melhora } \\
\text { Ausente } \\
\text { Presente } \\
\text { Ausente }\end{array}$ & $\begin{array}{l}\text { Melhora } \\
\text { Melhora } \\
\text { Ausente } \\
\text { Melhora } \\
\text { Ausente }\end{array}$ & $\begin{array}{l}\text { Melhora } \\
\text { Melhora } \\
\text { Ausente } \\
\text { Melhora } \\
\text { Ausente }\end{array}$ \\
\hline M & 47 & $\begin{array}{l}\text { Extração } \\
\text { glândula } \\
\text { lacrimal }\end{array}$ & $\begin{array}{l}\text { Canalículo } \\
\text { superior } \\
\text { ipsilateral à } \\
\text { glândula } \\
\text { retirada }\end{array}$ & $\begin{array}{l}\text { Sensação de corpo estranho } \\
\text { Ardor } \\
\text { Fotofobia } \\
\text { Hiperemia } \\
\text { Recanalização }\end{array}$ & $\begin{array}{l}\text { Mantida } \\
\text { Mantida } \\
\text { Ausente } \\
\text { Presente } \\
\text { Ausente }\end{array}$ & $\begin{array}{l}\text { Mantida } \\
\text { Mantida } \\
\text { Ausente } \\
\text { Presente } \\
\text { Ausente }\end{array}$ & $\begin{array}{l}\text { Mantida } \\
\text { Mantida } \\
\text { Ausente } \\
\text { Presente } \\
\text { Ausente }\end{array}$ & $\begin{array}{l}\text { Mantida } \\
\text { Mantida } \\
\text { Ausente } \\
\text { Melhora } \\
\text { Ausente }\end{array}$ & $\begin{array}{l}\text { Melhora } \\
\text { Melhora } \\
\text { Ausente } \\
\text { Melhora } \\
\text { Ausente }\end{array}$ \\
\hline
\end{tabular}

$\mathrm{Na}$ técnica descrita neste estudo a incisão engloba apenas o contorno da papila lacrimal e, por esta via, amplia-se a dissecção das paredes do canalículo, limitando-se apenas ao segmento vertical, propiciando menor trauma cirúrgico e recuperação mais rápida. A técnica de Putterman pode resultar na inversão da borda palpebral ${ }^{(9)}$, o que não se observou no presente estudo. Este fato poderia ser explicado pela incisão maior, provocando processo cicatricial mais intenso e retração da lamela posterior.

Dois dos casos aqui apresentados, assim como os daquele autor $^{(9)}$, foram submetidos à eletrocauterização do ponto lacrimal previamente à cirurgia. Tal modalidade terapêutica não é totalmente eficiente na oclusão permanente do ponto, ocorrendo alta taxa de recanalização ainda no primeiro mês, dependendo da profundidade que é inserido o cautério ${ }^{(3,10)}$ : nas cauterizações superficiais do ponto, $53 \%$; quando o cautério atinge $1,5 \mathrm{~mm}$ do canalículo vertical, $25 \%$ ou por toda sua extensão, $7 \%^{(3)}$. Os pontos tratados com cautério térmico podem permanecer fechados por um período de 28 semanas, comparados com aqueles cauterizados por laser (22 semanas) ${ }^{(10)}$. Quanto às complicações advindas da eletrocauterização, pode-se ter: distorção da margem palpebral próxima ao ponto, ou ao longo do canalículo, lesões palpebrais e fístulas, as quais comprometem o efeito oclusivo ${ }^{(3)}$. Portanto, além da pouca eficácia no fechamento definitivo do ponto lacrimal, pode, ainda, cursar com efeitos indesejáveis.

Alguns autores, em situações moderadas a severas de olho seco, preferem a oclusão do ponto, por meio de tampões de silicone ${ }^{(11-12)}$. No entanto, o sucesso de sua utilização é questionável. Em um dos estudos, de 22 olhos ocluídos com tam- pão, 82\% mostraram melhora clínica, não apresentando extrusão do material ${ }^{(12)}$. Porém, várias complicações foram descritas, como: deslocamento do tampão para as vias lacrimais acarretando dacriocistite, canaliculite e granuloma piogênico sobre o ponto, extrusão do mesmo e dissecção da mucosa canalicular por traumatismo mecânico ${ }^{(13-15)}$.

Outras tentativas de eliminar os sintomas da ceratoconjuntivite seca e de oferecer ao paciente uma melhor qualidade de vida, também foram sugeridas, com menor eficácia, como o uso da ciclosporina $\mathrm{A}^{(2)}$, colírios de metilprednisolona ${ }^{(8)}$, transplante autólogo da glândula submandibular ${ }^{(16)}$, soluções de palmitato de retinol ${ }^{(17)} \mathrm{e}$ injeção da toxina botulínica ${ }^{(18)}$ no canto medial, para diminuir a drenagem lacrimal.

Com tantos tratamentos propostos e nenhum totalmente eficiente, procurou-se criar uma técnica cirúrgica de oclusão do canalículo que pudesse manter a lágrima por mais tempo em contato com a superfície ocular, sem que houvesse a reabertura do mesmo. O acompanhamento dos pacientes deste estudo teve duração de seis meses e nenhum sinal de recanalização foi evidenciado. Maior casuística e seguimento serão necessários para que se confirme a validade deste procedimento.

\section{CONCLUSÃO}

Esta técnica, além de mostrar-se bastante efetiva e simples para a oclusão do canalículo lacrimal, não cursou com complicações, havendo melhora dos sintomas e permanecendo todos os pacientes com o canalículo fechado durante o período de seis meses. 


\section{ABSTRACT}

Purpose: To demonstrate the efficacy and possible complications of a surgical technique that includes the removal of the vertical portion of the lacrimal canaliculus in patients with dry eye syndrome. Methods: A study was performed on six canaliculi of six eyes (four patients). Three patients had dry eye, associated with primary Sjögren syndrome. One of the four patients developed keratoconjuntivitis sicca due to lacrimal gland removal. The criteria included: patients with symptoms of dry eye that did not improve even with the continuous use of artificial tears, low results (less than $5 \mathrm{~mm}$ ) with the Schirmer test, rose bengal staining and cases of recanalization after thermal occlusion with electrocauterium. The lacrimal puncta were examined after $7,15,30,60,90$ and 180 days after surgery. Results: None of the canaliculus recanalized at this time. Punctate keratitis, corneal filaments, Schirmer and rose bengal tests improved in 5 eyes. Difuse punctate keratitis was present in the patient with lacrimal gland removal even 2 months after of surgery. Eyelid margin complications were not observed. Conclusion: This technique is simple and effective in permanent lacrimal canaliculus occlusion. We did not note any complications compared to other procedures.

Keywords: Dry eye syndromes/surgery; Lacrimal apparatus/ surgery; Ophthalmologic surgical procedures/methods

\section{REFERÊNCIAS}

1. Scarpi JM. Olho seco. In: José NK, Belfort Junior R. Córnea - Clinica cirúrgica. São Paulo: Roca; 1997. p.285-99.
2. Kunert KS, Tisdale AS, Stern ME, Smith JA, Gipson IK. Analysis of topical cyclosporine treatment of patients with dry eye syndrome: effect on conjunctival lymphocytes. Arch Ophthalmol. 2000;118(11):1489-96.

3. Murube J, Murube E. Treatment of dry eye by blocking the lacrimal canaliculi. Surv Ophthalmol. 1996;40(6):463-80. Review.

4. Chibret H. Dry eyes syndrome: reality and perspectives of treatment. Ann Pharm Fr. 2002;60(2):115-22. Review. French.

5. Schein OD, Munoz B, Tielsch JM, Bandeen-Roche K, West S. Prevalence of dry eye among the elderly. Am J Ophthalmol. 1997;124(6):723-8.

6. Schaumberg DA, Buring JE, Sullivan DA, Dana MR. Hormone replacement therapy and dry eye syndrome. JAMA. 2001;286(17):2114-9.

7. Williamson $\mathrm{J}$. Investigating signs and symptoms in dry eyed patients. $\mathrm{Br} \mathrm{J}$ Ophthalmol. 1997;81(7):522.

8. Marsh P, Pflugfelder SC. Topical nonpreserved methylprednisolone therapy for keratoconjunctivitis sicca in Sjögren syndrome. Ophthalmology. 1999;106 (4):811-6.

9. Putterman AM. Canaliculectomy in the treatment of keratitis sicca. Ophthalmic Surg. 1991;22(8):478-80.

10. Vrabec MP, Elsing SH, Aitken PA. A prospective, randomized comparison of thermal cautery and argon laser for permanent punctal occlusion. Am J Ophthalmol. 1993;116(4):469-71.

11. Cohen EJ. Punctal occlusion. Arch Ophthalmol. 1999;117(3):389-90. Review.

12. Guzey M, Ozardali I, Kilic A, Basar E, Dogan Z, Satici A, Karadede S. The treatment of severe trachomatous dry eye with canalicular silicone plugs. Eye. 2001;15(Pt 3):297-303.

13. Rumelt S, Remulla H, Rubin PA. Silicone punctal plug migration resulting in dacryocystitis and canaliculitis. Cornea. 1997;16(3):377-9.

14. Akova YA, Demirhan B, Cakmakci S, Aydin P. Pyogenic granuloma: a rare complication of silicone punctal plugs. Ophthalmic Surg Lasers. 1999;30(7):584-5.

15. Fayet B, Assouline M, Hanush S, Bernard J, D'Hermies F, Renard G. Silicone punctal plug extrusion resulting from spontaneous dissection of canalicular mucosa: A clinical and histopathologic report. Ophthalmology. 2001; 108(2):405-9.

16. Geerling G, Sieg P, Meyer C, Bastian GO, Laqua H. Transplantation of autologous submandibular glands in very severe keratoconjunctivitis sicca. 2 year outcome. Ophthalmologe. 1998;95(4):257-65. Germany.

17. Kobayashi TK, Tsubota K, Takamura E, Sawa M, Ohashi Y, Usui M. Effect of retinol palmitate as a treatment for dry eye: a cytological evaluation. Ophthalmologica. 1997;211(6):358-61.

18. Sahlin S, Chen E, Kaugesaar T, Almqvist H, Kjellberg K, Lennerstrand G. Effect of eyelid botulinum toxin injection on lacrimal drainage. Am J Ophthalmol. 2000;129(4):481-6.

\section{Simpósio da Associação Paranaense de Oftalmologia}

\section{3 a 25 de Junho de 2005 Bourbon Curitiba Hotel - Curitiba - PR Tema Offial: Qualidade de Visão}

\section{INFORMAÇÕES: Creative Solution}

Tel: (11) 5575-0254 - Fax (11) 5539-2186

E-mail: congressoapo@ terra.com.br 